\title{
Average Household Income
}

National Cancer Institute

\section{Source}

National Cancer Institute. Average Household Income. NCI Thesaurus. Code C19308.

A demographic parameter indicating the average amount of earnings made by a family in a particular population or/and during a particular period of time. 